\title{
S100A9 is not essential for disease expression in an acute (K/BxN) or chronic (CIA) model of inflammatory arthritis
}

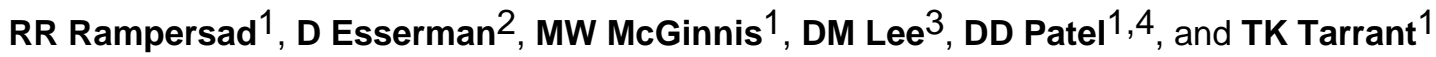 \\ ${ }^{1}$ Thurston Arthritis Research Center, University of North Carolina School of Medicine, Division of \\ Rheumatology, Allergy, and Immunology, Chapel Hill, NC ${ }^{2}$ University of North Carolina School of \\ Medicine, Division of General Medicine and Clinical Epidemiology, Chapel Hill, NC ${ }^{3}$ Division of \\ Rheumatology, Allergy, and Immunology, Brigham and Women's Hospital, Boston, MA, USA \\ ${ }^{4}$ Novartis Institutes for BioMedical Research, Basel, Switzerland
}

\begin{abstract}
Objective: S100A8 (calgranulin A, MRP8) and S100A9 (calgranulin B, MRP14) are calciumbinding proteins highly expressed by activated myeloid cells and thought to be involved in the pathogenesis of inflammatory diseases. Circulating levels of S100A8/S100A9 are elevated in both human and experimental models of autoimmune disease, including rheumatoid arthritis (RA).
\end{abstract}

Methods: Mice deficient in S100A9 (S100A9-/-) and wild-type controls were immunized using standard techniques for the $\mathrm{K} / \mathrm{BxN}$ serum transfer or the collagen-induced arthritis (CIA) model.

Results: S100A9-/- animals, with defective expression of both S100A8 and S100A9 proteins, had similar arthritis and histopathology to that of wild-type controls in both mouse models.

Conclusion: S100A8 and S100A9 are not essential for disease expression in either the $\mathrm{K} / \mathrm{BxN}$ serum transfer or the CIA model of inflammatory arthritis.

\begin{abstract}
Rheumatoid arthritis (RA) results in a dysregulated immune response that leads to inflammation and destruction of the synovial linings and joint structures. Crucial to this pathophysiological process are myeloid cells, including neutrophils, monocytes, and macrophages $(1,2)$, which can produce proinflammatory cytokines and matrix metalloproteinases leading to cartilage degradation and bone destruction (3). The collageninduced arthritis (CIA) and the K/BxN serum transfer models are two well-established mouse models of inflammatory arthritis that share many features with RA $(1,4)$. Both models involve inflammatory myeloid cells, develop erosions similar to human RA, and can be used to study the acute $(\mathrm{K} / \mathrm{BxN})$ and chronic (CIA) portions of the human disease $(1,4)$.

The S100 group of proteins is the largest group of calcium-binding proteins within the EFhand homology family (5). S100A8 (calgranulin A, MRP8) and S100A9 (calgranulin B, MRP14) are highly expressed in activated and circulating myeloid cells and form stable heterodimers that are secreted upon stimulation (5). Increased local and systemic protein expression has been linked to inflammatory diseases, including RA, since the 1980s $(2,5,6)$. Previous studies have implicated a role for S100A9/S100A8 in experimental inflammatory arthritis models $(2,7)$ and in human autoimmune-mediated diseases including RA $(2,6)$,
\end{abstract}

(C) 2009 Taylor \& Francis on license from Scandinavian Rheumatology Research Foundation

Teresa Tarrant, University of North Carolina at Chapel Hill, CB\# 7280, 3300 Manning Dr., Chapel Hill, NC 27599, USA.

tarra002@med.unc.edu 
Sjögren's syndrome, systemic lupus erythematosus, and inflammatory bowel disease [all reviewed in (5)].

The S100A9-deficient (S100A9-/-) mouse has undetectable protein levels of both S100A9 and S100A8 in peripheral neutrophils and monocytes/macrophages $(8,9)$, a deficit that has been attributed to the loss of S100A9-mediated regulation, which protects S100A8 from degradation (10). To examine the pathogenic role of S100A8/9 in inflammatory arthritis, we tested disease expression in S100A9-/- animals using the $\mathrm{K} / \mathrm{BxN}$ and CIA models of inflammatory arthritis.

\section{Materials and methods}

\section{Animals}

C57B1/6 and DBA-1J mice were either purchased from Jackson Laboratories (Bar Harbor, ME, USA) or bred and cared for in the Division of Laboratory Animal Medicine (DLAM) facilities under the approved The University of North Carolina Institutional Animal Care and Use Committee (IACUC) protocol number 06-242.0. The S100A9-deficient mice were provided by Dr E. Lorenz and previously generated by Dr W. Nacken and colleagues (8).

\section{$\mathrm{K} / \mathrm{BxN}$ arthritis model}

Male 6-week-old mice with a homozygous deficiency in S100A9 bred $>12$ generations onto the C57B1/6 background and wild-type C57B1/6 controls were injected on days 0 and 2 with $5 \mu \mathrm{L} \mathrm{K/BxN}$ serum per gram of mouse weight intraperitoneally, similar to previously published reports (1). Mice were measured for paw swelling, represented as a change in the mean thickness of the mouse's fore- and hindlimbs $(\mathrm{mm})$ from its baseline average, and a clinical index, which was scored by a blinded observer using the following system: $0=$ normal paw; $1=$ mild ankle or digit swelling; $2=$ moderate swelling of the ankle \pm any number of digits; 3 $=$ maximal swelling of the entire paw and digits. The maximum score per paw was 3 with a maximum score of 12 per mouse. At experiment termination, hindlimbs were fixed (4\% paraformaldehyde), decalcified, and embedded in paraffin for histopathology.

\section{Induction and evaluation of CIA}

S100A9-/- mice and wild-type controls were bred seven generations onto the susceptible DBA-1J background. Age-matched 8-10-week-old control and S100A9-/- mice were immunized with adjuvant (complete Freund's on day 0 and incomplete Freund's on day 21)

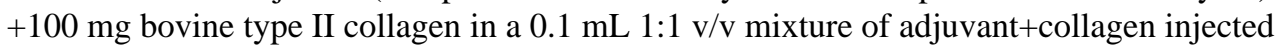
subcutaneously into the base of the tail, similar to previously published reports $(3,11)$. Mice were assessed by a blinded observer for paw swelling and clinical disease until experiment termination when hindlimbs were processed for histopathology, as described above.

\section{Histopathology}

Serial $5 \mu \mathrm{m}$ sections of paraffin-embedded tissue were cut and stained with haematoxylin and eosin (H\&E) according to standard protocols for morphological analysis.

\section{Data analysis}

Linear mixed models (random intercept and slope) were used to determine whether significant differences existed in the course of the disease over time (greatest order of magnitude was three) between the S100A9-deficient mice and wild-type controls. The overall group effect was assessed using a likelihood ratio test (LRT). Statistical analysis was performed using SAS version 9.1 . 


\section{Results}

\section{S100A9-deficient mice have similar disease to wild-type mice in the K/BxN serum transfer model}

$\mathrm{K} / \mathrm{BxN}$ serum transfer-mediated arthritis is a passive transfer model of autoantibodies recognizing glucose-6-phospate isomerase that leads to an acute, self-limited inflammatory arthritis (1). In this model, S100A9-/- mice had strikingly similar disease expression as measured by two independent criteria, paw swelling (Figure 1A) and clinical index (Figure 1B). There was no statistically significant difference between the S100A9-/- mice $(\mathrm{n}=10)$ and wild-type controls $(\mathrm{n}=12)$ in paw swelling ( $\mathrm{LRT}=3.5, \mathrm{df}=2, \mathrm{p}=0.174)$; however, the clinical index did have statistical significance despite extremely similar curves (LRT $=11.8$, $\mathrm{df}=2, \mathrm{p}=0.003$ ), which may have resulted from the minimal variability of this model and a tight standard error seen between the two groups. Consequently, histopathology was compared between S100A9-/- animals (Figure 1C) and wild-type controls (Figure 1D), and there was no apparent difference in the presence or severity of joint damage or bony erosions.

\section{S100A9-deficient mice have minimally increased disease compared to wild-type mice in the CIA model}

To determine whether S100A9-/- animals had a difference in a chronic, de novo inflammatory arthritis disease model, we studied them in CIA. Of note, S100A9-/- animals $(\mathrm{n}=18)$ had mildly increased disease scores compared to wild-type controls $(n=15)$ in both paw swelling (Figure 2A; LRT $=13.4, \mathrm{df}=4, \mathrm{p}=0.010$ ) and clinical index (Figure 2B; $\mathrm{LRT}=8.1, \mathrm{df}=4$, $\mathrm{p}=0.088$ ), but only paw swelling achieved statistical significance. However, the

histopathology between the two groups showed that there was similar joint damage and bony erosions (Figure 2C and 2D) despite mildly different disease curves.

\section{Discussion}

The S100A9/S100A8 heterodimer has been identified in the inflamed synovial tissue of RA patients (6), and increased levels in serum samples and synovial fluid have correlated with disease activity $(5,12)$. Indeed, several investigators have suggested this heterodimer as a potential biomarker of disease activity in inflammatory arthritis and other autoimmune disease states where myeloid cells play a crucial role $(5,13)$.

van Lent et al recently described decreased joint inflammation and cartilage destruction from methylated bovine serum albumin (mBSA) antigen-induced arthritis in S100A9-deficient mice (2). Similar to our study, S100A9-/- animals were capable of developing arthritis. In addition, outcome measures of cellular inflammation in the S100A9-/- joints did not differ significantly at some time points (2). The mBSA model differs from both $\mathrm{K} / \mathrm{BxN}$ and CIA in that a booster injection is administered locally into the joint, which leads to a chronic destructive localized arthritis, whereas $\mathrm{K} / \mathrm{BxN}$ is a systemic antibody transfer and CIA is a de novo-induced immune response dependent on the $\mathrm{H}-2^{\mathrm{q}}$ haplotype (4). These technical, and perhaps other mechanistic, differences may additionally explain the differing results observed between the mBSA model and our studies.

Despite the absence of S100A9 or S100A8 protein expression in the S100A9-/- mouse, myeloid cells have normal morphology, in vivo leucocyte migration, phagocytosis, and respiratory burst $(8,9)$. Our data also suggest that the functional roles of myeloid cells in the $\mathrm{K} / \mathrm{BxN}$ and CIA models are preserved, despite loss of S100A9/S100A8 protein levels, because only small clinical and no histopathological differences were seen between groups. Although statistical significance was achieved in one of two clinical measures in both arthritis models, the disease course, similarity in joint erosions, and inherent variability of the models would 
argue that the overall disease outcome was not dramatically affected by the absence of these two proteins.

It is possible that either compensatory or overlapping mechanisms are at play in the S100A9 $-1-$ mouse, which could explain why larger differences were not seen between the experimental and control groups. Specifically, S100A12, a proinflammatory calgranulin with high expression within myeloid cells, has also been linked to RA and psoriatic arthritis $(5,14)$. Additionally, S100A4 is highly expressed in RA synovial tissue and synovial fluid and may play a significant role in the pathogenesis of chronic inflammation and joint destruction (15). Alternatively, either other proinflammatory mediators could compensate for S100A9/S100A8 deficiency or the heterodimer may play a less significant role in mouse models of inflammation than in human disease.

\section{Acknowledgments}

We thank Dr David Siderovski for critical review of the manuscript and Dr Eva Lorenz for providing the S100A9deficient animals. We also acknowledge receipt of an American College of Rheumatology Physician Scientist Award and NIH K08 AI070684-01 for funding.

\section{References}

1. Kamradt T, Schubert D. The role and clinical implications of G6PI in experimental models of rheumatoid arthritis. Arthritis Res Ther 2005;7:20-8. [PubMed: 15642150]

2. van Lent PL, Grevers L, Blom AB, Sloetjes A, Mort JS, Vogl T, et al. Myeloid-related proteins S100A8/ S100A9 regulate joint inflammation and cartilage destruction during antigen-induced arthritis. Ann Rheum Dis 2008;67:1750-8. [PubMed: 18055478]

3. Lee SW, Kim JH, Park MC, Park YB, Lee SK. Adiponectin mitigates the severity of arthritis in mice with collagen-induced arthritis. Scand J Rheumatol 2008;37:260-8. [PubMed: 18612926]

4. Wooley PH, Luthra HS, Stuart JM, David CS. Type II collagen-induced arthritis in mice. I. Major histocompatibility complex (I region) linkage and antibody correlates. J Exp Med 1981;154:688-700. [PubMed: 6792316]

5. Foell D, Frosch M, Sorg C, Roth J. Phagocyte-specific calcium-binding S100 proteins as clinical laboratory markers of inflammation. Clin Chim Acta 2004;344:37-51. [PubMed: 15149869]

6. Odink K, Cerletti N, Bruggen J, Clerc RG, Tarcsay L, Zwadlo G, et al. Two calcium-binding proteins in infiltrate macrophages of rheumatoid arthritis. Nature 1987;330:80-2. [PubMed: 3313057]

7. Zenz R, Eferl R, Kenner L, Florin L, Hummerich L, Mehic D, et al. Psoriasis-like skin disease and arthritis caused by inducible epidermal deletion of Jun proteins. Nature 2005;437:369-75. [PubMed: 16163348]

8. Manitz MP, Horst B, Seeliger S, Strey A, Skryabin BV, Gunzer M, et al. Loss of S100A9 (MRP14) results in reduced interleukin-8-induced $\mathrm{CD} 11 \mathrm{~b}$ surface expression, a polarized microfilament system, and diminished responsiveness to chemoattractants in vitro. Mol Cell Biol 2003;23:1034-43. [PubMed: 12529407]

9. Hobbs JA, May R, Tanousis K, McNeill E, Mathies M, Gebhardt C, et al. Myeloid cell function in MRP-14 (S100A9) null mice. Mol Cell Biol 2003;23:2564-76. [PubMed: 12640137]

10. Vogl T, Ludwig S, Goebeler M, Strey A, Thorey IS, Reichelt R, et al. MRP8 and MRP14 control microtubule reorganization during transendothelial migration of phagocytes. Blood 2004;104:42608. [PubMed: 15331440]

11. Kurosaka D, Yoshida K, Yasuda J, Yasuda C, Noda K, Furuya K, et al. The effect of endostatin evaluated in an experimental animal model of collagen-induced arthritis. Scand J Rheumatol 2007;36:434-41. [PubMed: 18092264]

12. Sunahori K, Yamamura M, Yamana J, Takasugi K, Kawashima M, Yamamoto H, et al. The S100A8/ A9 heterodimer amplifies proinflammatory cytokine production by macrophages via activation of nuclear factor kappa B and p38 mitogen-activated protein kinase in rheumatoid arthritis. Arthritis Res Ther 2006;8:R69. [PubMed: 16613612] 
13. Nacken W, Roth J, Sorg C, Kerkhoff C. S100A9/S100A8: myeloid representatives of the S100 protein family as prominent players in innate immunity. Microsc Res Tech 2003;60:569-80. [PubMed: 12645005]

14. Foell D, Kane D, Bresnihan B, Vogl T, Nacken W, Sorg C, et al. Expression of the pro-inflammatory protein S100A12 (EN-RAGE) in rheumatoid and psoriatic arthritis. Rheumatology (Oxford) 2003;42:1383-9. [PubMed: 12832707]

15. Klingelhofer J, Senolt L, Baslund B, Nielsen GH, Skibshoj I, Pavelka K, et al. Up-regulation of metastasis-promoting S100A4 (Mts-1) in rheumatoid arthritis: putative involvement in the pathogenesis of rheumatoid arthritis. Arthritis Rheum 2007;56:779-89. [PubMed: 17328050] 

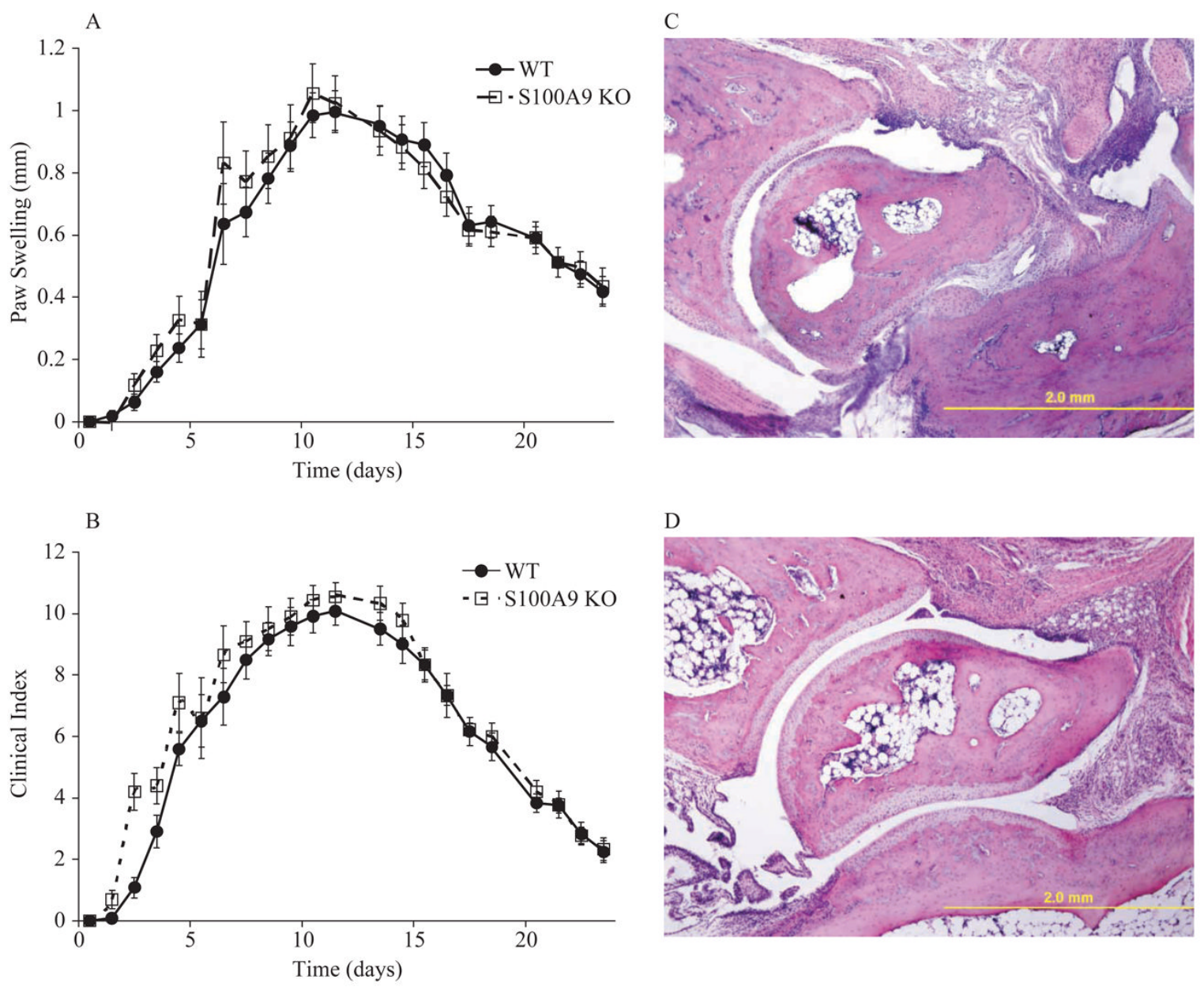

Figure 1.

S100A9-deficient mice have a similar disease course to wild-type (WT) mice in the K/BxN serum transfer model. The data represents 22 animals from two independent experiments. Male 6-week-old mice with a homozygous deficiency in S100A9 (S100A9-/-) (n=10) and WT controls $(n=12)$ were injected on days 0 and 2 with $5 \mu \mathrm{L} \mathrm{K} / \mathrm{BxN}$ serum per gram of mouse weight intraperitoneally. (A) Paw swelling was represented as a change in the mean thickness of the mouse's fore- and hindlimbs ( $\mathrm{mm})$ from its baseline average, and there was no difference between S100A9-/- and WT controls (LRT = 3.5, df = 2, p = 0.174). (B) Clinical disease assessment was scored from 0 to 3 by a blinded reader $(\mathrm{LRT}=11.8, \mathrm{df}=2, \mathrm{p}=0.003)$. Representative histopathology from (C) an S100A9-/- animal and (D) a WT control shows similar erosive disease. 


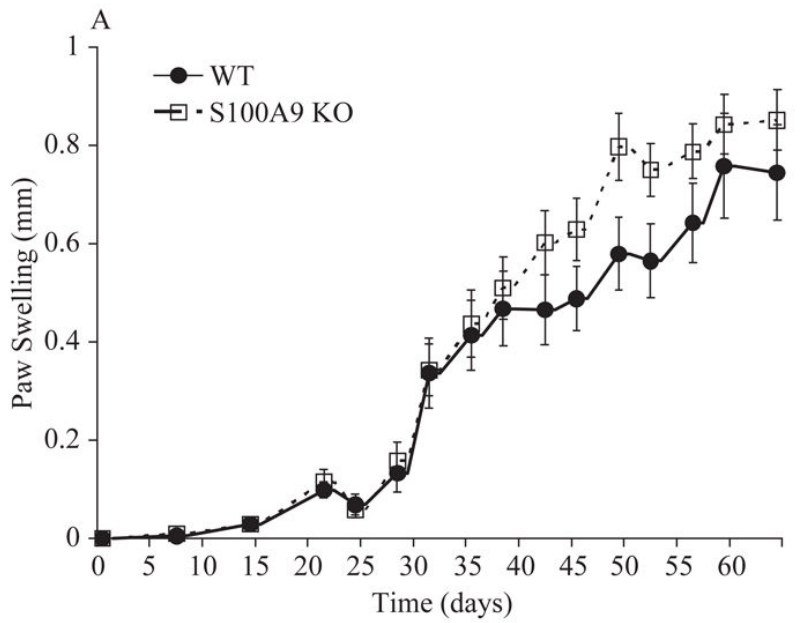

C

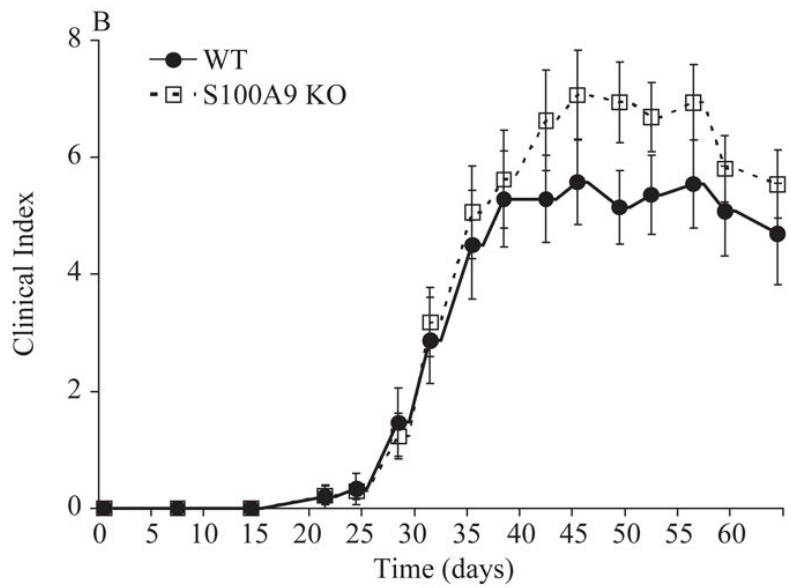

D

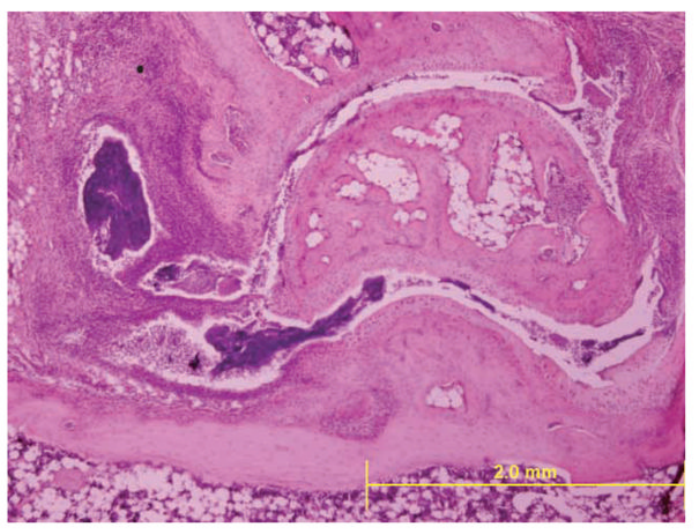

Figure 2.

S100A9-deficient mice have minimally increased disease compared to wild-type (WT) mice in the CIA model. The data represent 33 animals from two independent experiments. Agematched 8-10-week-old S100A9-/- mice $(n=18)$ and WT controls $(n=15)$ were immunized with adjuvant (complete Freund's on day 0 and incomplete Freund's on day 21) $+100 \mu \mathrm{g}$ bovine type II collagen. (A) Paw swelling was represented as a change in the mean thickness of the mouse's fore- and hindlimbs (mm) from its baseline average. S100A9-/- animals had mildly increased paw swelling compared to WT controls ( $\mathrm{LRT}=13.4, \mathrm{df}=4, \mathrm{p}=0.010)$. (B) Clinical disease was also mildly increased, but this was not statistically significant $(\mathrm{LRT}=8.1, \mathrm{df}=4$, $\mathrm{p}=0.088$ ). Representative histopathology from (C) an S100A9-/- animal and (D) a WT control shows similar erosive disease. 